\title{
MCKAY'S CORRESPONDENCE AND CHARACTERS OF FINITE SUBGROUPS OF SU(2)
}

\author{
W. ROSSMANN
}

\begin{abstract}
According to McKay [1980] the irreducible characters of finite subgroups of SU(2) are in a natural 1-1 correspondence with the extended Coxeter-Dynkin graphs of type ADE. We show that the character values themselves can be given by an uniform formula, as special values of polynomials which arise naturally as numerators of Poincaré series associated to finite subgroups of $\mathrm{SU}(2)$ acting on polynomials in two variables. These polynomials have been the subject of a number of investigations, but their interpretation as characters has apparently not been noticed.
\end{abstract}

\section{INTRODUCTION}

In 1980 McKay announced his astounding discovery that the finite subgroups of $\mathrm{SU}(2)$ are in natural 1-1 correspondence with the extended Coxeter-Dynkin graphs of type ADE in the following way. Let $K$ be a finite subgroup of $\mathrm{SU}(2),\left\{\chi_{i}\right\}$ its irreducible characters, and $\chi$ the character of its natural representation on $\mathbb{C}^{2}$. Let $M=\left(m_{i j}\right)$ be the matrix defined by

$$
\chi \chi_{i}=\sum_{j} m_{i j} \chi_{j} .
$$

The matrices $M$ corresponding to the finite subgroups of $S U(2)$ exactly the matrices of the form $M=2 I-C$ where $C$ is the Cartan matrix of an extended Coxeter-Dynkin graph of type ADE. McKay apparently found and verified this fact by direct computation. In the meantime there have been many attempts to explain it in other ways or to provide further insight into this phenomenon. Steinberg offered an explanation in terms of representation theory of finite groups in 1982 . Gonzales-Sprinberg and J.-L. Verdier [1983] gave an explanation in terms of algebraic geometry, an approach also taken up by Knörrer [1985]. Kostant [1985] found a remarkable relation between certain Poincaré polynomials associated to these groups and the action of the Coxeter element on the ADE root system, and Springer [1987] gave another method for the computation of these polynomials. They play an important role in all papers mentioned, except for Steinberg's. Mysteriously, they also appeared in an entirely different context in a paper of Lusztig in [1983] and reappeared in his paper in [1999]. It turns out that they have another striking feature, also rather strange at first sight: these polynomials 'are' the irreducible characters of the finite subgroups of $\mathrm{SU}(2)$, if this statement is taken with a grain of salt. The precise formulation is given in Theorem 3.1, the proof in $\S 4$. Some of the constructions in the proof have parallels in Galois theory of algebraic number fields having as common origin a method of localization for Galois groups at prime ideals (Lemmas 4.1,4.2): here the Galois group is $K$ and its localization is a maximal abelian subgroup $T$. The ADE graphs of $\S 2$ encode those properties 
the configuration of the these $T$ s inside of $K$ which are needed in Theorem 3.1 to identify the polynomials as characters.

\section{THE GRAPHS}

Let $V$ be a two dimensional complex vector with a unitary inner product. Let $G$ be a finite subgroup of its unitary group $\mathrm{U}(V)$, and let $Z=G \cap \mathrm{U}(\mathbb{C})$ its intersection with the center of $\mathrm{U}(V)$. We shall be concerned with maximal abelian subgroups of $G$, typically denoted $H$ with normalizer $N(H)$.

Lemma 2.1. (a)Any two maximal abelian subgroups $H$ of $G$ intersect in $Z$ only.

(b) The groups $W=N(H) / H$ have order 1 or 2 .

This is immediate from the observation that any non-scalar element of $\mathrm{U}(V)$ has two distinct eigenvalues, but it will be useful to keep in mind how this happens. Each maximal abelian subgroup $H$ of $G$ corresponds to a pair of lines $P, P^{-}$, its eigenspaces. The $P$ s which arise in this way are the singular lines, invariant under some non-scalar element in $G$. Let $\mathcal{P}$ denote the set of these $P$ s, a subset of $\mathbb{P}(V)$. Two $H$ s are $G$-conjugate if and only if the corresponding (unordered) pairs $\left\{P, P^{-}\right\}$ are. A conjugacy class of $H \mathrm{~s}$ corresponds to either 1 or 2 orbits of $P \mathrm{~s}$, depending on whether $P$ and $P^{-}$belong to the same orbit or not. In the first case $N(H) / H$ has order 2 and interchanges $P$ and $P^{-}$; in the second case $N(H) / H$ has order 1 . The map $G / H \rightarrow G / N(H)$ is $2: 1$ in the first case, 1:1 in the second. In the first case, when the orbits of $P$ and $P^{-}$coincide, we call this orbit doubled, in the second case, when they are different, we call these orbits coupled. The same terminology applies to the corresponding class of $H$ s. The decomposition of $G$ into conjugacy classes then gives

$$
|\bar{G}|=1+\frac{|\bar{G}|}{|N(\bar{H})|}(|\bar{H}|-1)+\cdots
$$

where $\bar{G}=G / Z, \bar{H}=H / Z$, and the sum is extended over a complete set of representatives for the conjugacy classes of $H$ s. The equation is the class equation for these groups. Its solution for the possible values of $|\bar{H}|,|N(\bar{H})|,|\bar{G}|$ is goes back to the beginnings of the theory, as does the determination of the groups themselves.

The finite subgroups $\bar{G}$ of $\mathrm{U}(V) / \mathrm{U}(\mathbb{C}) \approx \mathrm{PSU}(2) \approx \mathrm{SO}(3)$ are the polyhedral groups. We list them under three headings ADE in the notation of [Coxeter, 1974, p15].

Type A. $\bar{G}=(p) \approx \mathfrak{C}_{p}$, cyclic of order $p, p=1,2 \cdots$

Type D. $\bar{G}=(p, 2) \approx \mathfrak{D}_{p}$, dihedral of order $2 p ., p=1,2 \cdots$

Type E.

Tetrahedral: $\bar{G}=(3,3,3) \approx \mathfrak{A}_{4}$, alternating of order 12 ,

Octahedral: $\bar{G}=(4,3,2) \approx \mathfrak{S}_{4}$, symmetric of order 24 ,

Icosahedral: $\bar{G}=(5,3,2) \approx \mathfrak{A}_{5}$, alternating of order 60 .

The notation $\bar{G}=(5,3,2)$, for example, means that there are three singular orbits of this group on $\mathbb{P}(V) \approx \mathbb{P}^{1} \approx \mathbb{S}^{2}$ with stabilizers $\bar{H}$ of orders $5,3,2$, the three cyclic groups of rotations about three axes of symmetries of the icosahedron passing through a vertex, face, or edge.

Of particular interest are the finite subgroups of $\mathrm{SU}(V)$, which are known as binary polyhedral groups or Klein groups. We use the letters $K, T$ instead of $G, H$ for these. Except for odd cyclic groups, they are all inverse images of polyhedral 
groups under the map $\mathrm{SU}(V) \rightarrow \mathrm{SO}(3)$ with kernel $\{ \pm 1\}$ which here replaces the map $\mathrm{U}(V) \rightarrow \mathrm{SO}(3)$ with kernel $\mathrm{U}(\mathbb{C})$.

It is convenient to use the isomorphism of $\mathrm{SU}(2)$ with the group $\mathrm{U}(\mathbb{H})$ of quaternions of norm one to represent $K$ as a subgroup of $\mathrm{U}(\mathbb{H})$. For this purpose $\mathbb{H}$ is considered as a left vector space of $\mathbb{C}$ via $\sqrt{-1} x=i x$ with $i^{2}=-1$ in $\mathbb{H}$. $V$ is taken to be $\mathbb{H}$ equipped with this complex structure. The action of $U(\mathbb{H})$ on $\mathbb{H}$ by right multiplication gives its identification with $\mathrm{U}(V)$, written $u \cdot x=x u^{-1}$ in order to have $\mathrm{U}(V)$ act on the left, as usual. Three generators $e_{A}, e_{B}, e_{C}$ for $K$ may be chosen in the form

$$
e_{A}=e^{\frac{\pi}{p_{A}} J_{A}}, \quad e_{B}=e^{\frac{\pi}{p_{B}} J_{B}}, \quad e_{C}=e^{\frac{\pi}{p_{C}} J_{C}}
$$

for suitable quaternions $J_{A}, J_{B}, J_{C}$ satisfying $J^{2}=-1$ [Coxeter 1974, p68]. The three cyclic subgroups $T_{A}, T_{B}, T_{C}$ generated by $e_{A}, e_{B}, e_{C}$ are the isotropy groups at three base points $P_{A}, P_{B}, P_{C}$ for the three singular orbits $\mathcal{P}_{A}, \mathcal{P}_{B}, \mathcal{P}_{C}$ of $K$ on $\mathbb{P}(V)$. The graphs below are designed to encode some properties the configuration of the singular lines $P_{A}, P_{B}, P_{C}$ in $V$ through the configuration of their stabilizers $T_{A}, T_{B}, T_{C}$ inside of $K$, as explained in the legend.

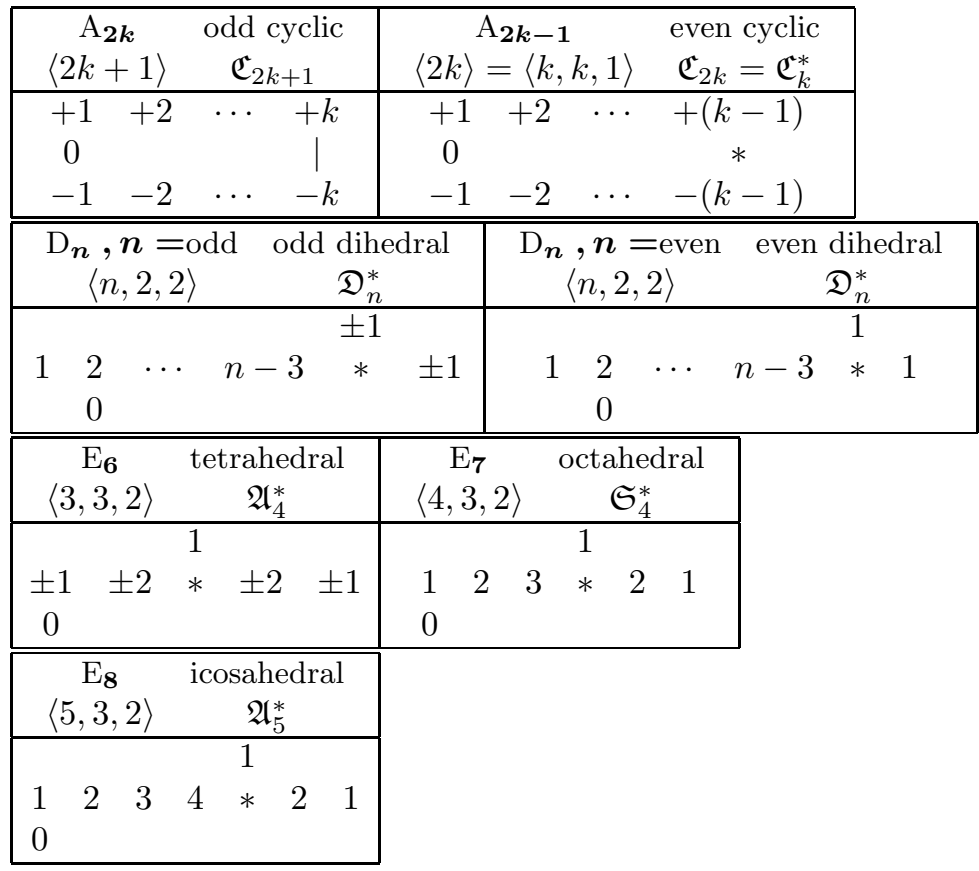

Legend. The first line lists the polyhedral type, the second a symbol for $K$ like $\langle 5,3,2\rangle$ and a name for it as abstract group like $\mathfrak{A}_{5}^{*}$; the star indicates the extension by $\{ \pm 1\}$ via $\mathrm{SU}(2) \rightarrow \mathrm{SO}(3)$. The cyclic case of type $\mathrm{A}$, will be omitted throughout, as it would only contribute some awkward complications in terminology. Each graph consists of three branches, strings of nodes joined at a central node labeled $*$. We use the letters $A, B, C$ as labels for the three branches and various items attached to these.

The three branches of the graph correspond to the three singular orbits $\mathcal{P}_{A}, \mathcal{P}_{B}, \mathcal{P}_{C}$ of $K$ on $\mathcal{P}$, its three singular orbits on $\mathbb{P}(V)$. The isotropy groups are cyclic and for an appropriate choice of base points $P_{A}, P_{B}, P_{C}$ they can be taken to be the three 
cyclic subgroups $T_{A}, T_{B}, T_{C}$ corresponding to the three generators $e_{A}, e_{B}, e_{C}$ of $K$ mentioned above. These three subgroups form a complete system of representatives for the maximal abelian subgroups of $K$, but redundant for odd $\mathrm{D}_{n}$ and for $\mathrm{E}_{6}$.

The elements of $T_{A}=\left\{e_{A}^{ \pm n}=\exp \left( \pm n \frac{\pi}{p_{A}} J_{A}\right), n=1, \cdots, p_{A}\right\}$ are represented by the nodes labeled $n$ or $\pm n$, to be specified as $n A$ or $\pm n A$ to indicate the branch $A$, if necessary. Each node, whether labeled $n$ or $\pm n$, represents two elements $\exp \left( \pm n \frac{\pi}{p} J\right)$, except when these coincide. A branch on which the label is $n$ the group $T$ represents a doubled class in the sense explained above; branches on which the label is $\pm n$ come in pairs, say $A, B$, and the corresponding groups $T_{A}, T_{B}$ are coupled, hence conjugate within $K$. The two branches $A, B$ are then related by a symmetry of the graph, which occurs only for odd $\mathrm{D}_{n}$ and for $\mathrm{E}_{6}$ and the four elements $\exp \left( \pm n \frac{\pi}{p_{A}} J_{A}\right), \exp \left( \pm n \frac{\pi}{p_{B}} J_{B}\right)$ lie in two conjugacy classes, each containing two of the elements, namely the two corresponding to the choices $(+,-)$ or $(-,+)$ of the ambiguous signs $( \pm, \pm)$ : opposite signs correspond to conjugate elements, equal signs to non-conjugate elements. The inversion involution $[c] \mapsto\left[c^{-1}\right]$ on conjugacy classes interchanges coupled classes and fixes all others, and is therefore represented by the symmetry of the graph which interchanges coupled nodes on symmetric branches.

The central node labeled $*$ corresponds to the central element -1 of $K$ inside of $\mathrm{SU}(2)$ and lies in all $T \mathrm{~s}$. The node labeled 0 may stand for the identity element and with this interpretation it should be thought of as attached to the ends of all branches; but it has been placed next to a particular node so as to produce the usual extended Coxeter-Dynkin graph, if taken as attached to that node [Bourbaki, 1968]. This extra node is added so that the graph may do double duty as McKay's character graph, associated to the Cartan matrix in the way explained in the introduction. In this interpretation the extra node stands for the trivial character. Detailed verifications are omitted, except for the following lemma, which justifies most of the rules given above.

Lemma 2.2. Two coupled nodes $\pm n, \pm n$ represent two conjugacy classes. Each pair with opposite signs represents the same class, each pair with equal signs represents inverse classes.

Proof. The coupling on nodes can occur only for $\mathrm{D}_{n}$ and for $\mathrm{E}_{6}$, where the assertion be verified directly. For $\mathrm{E}_{6}$, when $K=\langle 3,3,2\rangle$, the three vertices can be taken to be [Coxeter, 1974, p76]

$$
J_{A}=\frac{1}{\sqrt{3}}(i+j+k), J_{B}=\frac{1}{\sqrt{3}}(i-j+k), J_{C}=i .
$$

One has $e_{C}=e^{\frac{\pi}{2} i}=i$ and $i J_{A} i^{-1}=-J_{B}$. Hence $e_{C} e_{A} e_{C}^{-1}=e_{B}^{-1}$. This shows that conjugation by $e_{C}$ implements the coupling of the two branches $A, B$ as indicated in the graph. For $\mathrm{D}_{n}$ the verification is similar.

The reflection group $\boldsymbol{K}^{\prime}$. To start with, let $K \subset G$ be any 'normal extension' of $K \subset \mathrm{SU}(V)$ to a finite group $G \subset \mathrm{U}(V)$. Then $\bar{K} \subset \bar{G}$ is a normal extension of polyhedral groups in $\mathrm{SO}(3)$. (The few possibilities are well-known [Coxeter, $1974, \S 7.1-7.3]$, but will not be needed here.) The three singular orbits of $K$ and $G$ on $\mathbb{P}(V)$ depend only on $\bar{K}$ and $\bar{G}$. In particular, if $\bar{G}=\bar{K}$ then $G$ has the same singular $P$ s as $K$, hence the maximal abelian groups $H$ of $G$ are extensions of the maximal abelian groups $T$ on $K$. Generally, $G$ permutes the singular $P$ s 
of $K$ on $\mathbb{P}(V)$ and $G / K$ permutes the three $K$-orbits of these $P$ s. The $K$-orbits corresponding to branches of the same length are permuted among themselves, so that this action of $G / K$ must be trivial except for $\mathrm{D}_{n}$ and $\mathrm{E}_{6}$. On the other hand, if $G / K$ leaves a singular $K$-orbit invariant, then this situation is represented in the form $s P=P$ for some $s \in G$ and some $P$ in this orbit. Similar remarks apply to the action of $G / K$ on the conjugacy classes of $T$ s. Consider in particular the situation when one of the three $T \mathrm{~s}$ for $K$ is invariant by some $s \in G$, i.e. $s T s^{-1}=T$. In that case $s$ permutes the two invariant lines $P, P^{-}$of $T$, leading to two cases, as indicated.

$$
\begin{aligned}
& \text { Case (1). } s \cdot P=P, s \cdot P^{-}=P^{-} . T:\left(\begin{array}{cc}
\lambda & 0 \\
0 & \lambda^{-}
\end{array}\right), s=\left(\begin{array}{cc}
\mu & 0 \\
0 & \mu^{-}
\end{array}\right) . \\
& \text {Case }(2) s \cdot P=P^{-}, s \cdot P^{-}=P . T:\left(\begin{array}{cc}
\lambda & 0 \\
0 & \lambda^{-}
\end{array}\right), s=\left(\begin{array}{cc}
0 & \sigma \\
1 & 0
\end{array}\right) .
\end{aligned}
$$

Case (1) occurs whenever an element $s$ of $G$ leaves invariant a singular line $P$ of $K$. If $s$ is furthermore a reflection, i.e. leaves pointwise fixed a subspace of codimension 1 , then exactly one of $\mu, \mu^{-}$must be $=1$.

It is a remarkable fact that each finite subgroup $K$ of $\mathrm{SU}(2)$ is contained with index 2 in a subgroup of $\mathrm{U}(2)$ generated by three reflections of order 2 [Coxeter, 1974, p93]. The reflection group associated to $K=\left\langle p_{A}, p_{B}, p_{C}\right\rangle$ in this way is denoted $K^{\prime}=\left\langle p_{A}, p_{B}, p_{C}\right\rangle^{\prime}$. In this case the action of the two element group $K^{\prime} / K$ on the three singular orbits $\mathcal{P}_{A}, \mathcal{P}_{B}, \mathcal{P}_{C}$ gives an involution on the graph encoding these orbits. The following lemma identifies this action of $K^{\prime} / K$ as the inversion involution $[c] \mapsto\left[c^{-1}\right]$ on conjugacy classes.

Lemma 2.3. $K^{\prime} / K$ acts by inversion on the conjugacy classes in $K$.

Proof. It suffices to show that $K^{\prime} / K$ acts by inversion on the classes of the three generators $e_{A}, e_{B}, e_{C}$ of the three cyclic groups. In any case $K^{\prime} / K$ must map the class of one of these generator to a class of the same order. Thus only coupled end-nodes $\pm 1, \pm 1$ need be considered, for odd $\mathrm{D}_{n}$ and for $\mathrm{E}_{6}$. Take the latter case, for example.

From the construction in [Coxeter, 1974, §9.5] the group $K^{\prime}=\langle 3,3,2\rangle^{\prime}$ has as the two element group $\langle 4,3,2\rangle /\langle 3,3,2\rangle$ as a quotient and the action of $K^{\prime} / K$ on the classes in $K=\langle 3,3,2\rangle$ agrees with that of $\langle 4,3,2\rangle /\langle 3,3,2\rangle$. Thus it remains to check that $\langle 4,3,2\rangle /\langle 3,3,2\rangle$ acts by inversion on the classes in $\langle 3,3,2\rangle$. It follows from the construction of $\langle 4,3,2\rangle$ from $\langle 3,3,2\rangle$ in [Coxeter, 1974, $\S 7.3$ ] that $\langle 4,3,2\rangle$ has an element which interchanges the two generators $e_{A}$ and $e_{B}$ of $\langle 3,3,2\rangle$. The classes $e_{A}$ and $e_{B}$ in $\langle 3,3,2\rangle$ are indeed inverses of each other, as can be read off the $\mathrm{E}_{6}$ graph and has been verified in the proof of the preceding lemma.

Four groups associated to a Schwarz triangle. We mention a few facts about the groups under consideration, but the further development is logically independent of these. To get an overview it seems best to start with a Schwarz triangle of the first kind, a triangle on a 2 -sphere, with vertices $A, B, C$ whose angles $\pi / p_{A}, \pi / p_{B}, \pi / p_{C}$ satisfy the inequality

$$
\frac{1}{p_{A}}+\frac{1}{p_{B}}+\frac{1}{p_{C}}>1
$$

There are four groups associated to such a Schwarz triangle. They may be described as follows. The groups in question are transformation groups on a real or complex 
space $V_{\mathbb{R}}$ or $V_{\mathbb{C}}$, copies of $\mathbb{R}^{3}$ or $\mathbb{C}^{2}$. We denote them $K_{\mathbb{R}}, K_{\mathbb{R}}^{\prime}, K_{\mathbb{C}}, K_{\mathbb{C}}^{\prime} \cdot K_{\mathbb{R}}$ and $K_{\mathbb{C}}$ are finite subgroups of $\mathrm{O}\left(V_{\mathbb{R}}\right)$ and $\mathrm{U}\left(V_{\mathbb{C}}\right), K_{\mathbb{R}}^{\prime}$ and $K_{\mathbb{C}}^{\prime}$ are reflection groups in $\mathrm{O}\left(V_{\mathbb{R}}\right)$ and $\mathrm{SU}^{\prime}\left(V_{\mathbb{C}}\right)$ containing $K_{\mathbb{R}}$ and $K_{\mathbb{C}}$ as subgroups of index two. $\left(\mathrm{SU}^{\prime}\left(V_{\mathbb{C}}\right)\right.$ is the group generated by $\mathrm{SU}\left(V_{\mathbb{C}}\right)$ together with $i$ and consists of unitary matrices of determinant \pm 1 .)

Realize the 2 -sphere carrying the triangle inside the 3 -space of quaternions $x \in \mathbb{H}$ satisfying $\bar{x}=-x$. It is then given by the equations $\bar{x}=-x, x \bar{x}=1$, which may be combined into $x^{2}=-1$. Write $J_{A}, J_{B}, J_{C}$ for the vertices $A, B, C$ considered as quaternions. $V_{\mathbb{R}}$ or $V_{\mathbb{C}}$ are realized in terms of quaternions as indicated below, a quaternion $i$ satisfying $i^{2}=-1$ being required for $V_{\mathbb{C}}$. The groups have generators $t=t_{A}, t_{B}, t_{C}$ and $s=s_{A}, s_{B}, s_{C}$ which act on $x \in \mathbb{H}$ through certain elements $e=e_{A}, e_{B}, e_{C}$ and $u=u_{A}, u_{B}, u_{C}$ as indicated. The generators are determined by the triangle $\left(e_{A}=\exp \left(\pi J_{A} / p_{A}\right), u_{A}=\exp \left(\frac{\pi}{2} J_{B C}\right)=J_{B C}:=\left[J_{B}, J_{C}\right] /\left\|\left[J_{B}, J_{C}\right]\right\|\right)$ and the relations satisfied by them can be extracted from [Coxeter, 1974]. The data are summarized in the tables below. The labels $\left(p_{A}, p_{B}, p_{C}\right)$ and $\left\langle p_{A}, p_{B}, p_{C}\right\rangle$ for these groups are those of Coxeter. Cyclic permutations of $A, B, C$ in the relations written out are understood.

\begin{tabular}{|c|c|c|c|c|}
\hline \multicolumn{3}{|c|}{$V_{\mathbb{R}}: \bar{x}=-x$} & generators & relations(\&cyclic) \\
\hline$\overline{\mathrm{SO}\left(V_{\mathbb{R}}\right)}$ & $K_{\mathbb{R}}$ & $\left(p_{A}, p_{B}, p_{C}\right)$ & $t: e x e^{-1}$ & $t_{A}^{p_{A}}=\left(t_{A} t_{B} t_{C}\right)=1$ \\
\hline $\mathrm{O}\left(V_{\mathbb{R}}\right)$ & $\overline{K_{\mathbb{R}}^{\prime}}$ & $\left(p_{A}, p_{B}, p_{C}\right)^{\prime}$ & $s: u \bar{x} u^{-1}$ & $s_{A}^{2}=1,\left(s_{A} s_{B}\right)^{p_{C}}=1$ \\
\hline \multicolumn{3}{|c|}{$V_{\mathbb{C}}: \sqrt{-1} x:=i x$} & generators & $\begin{array}{c}\text { relations (\&cyclic) } \\
\end{array}$ \\
\hline$\overline{\mathrm{SU}\left(V_{\mathbb{C}}\right)}$ & $K_{\mathbb{C}}$ & $\left\langle p_{A}, p_{B}, p_{C}\right\rangle$ & $t: x e^{-1}$ & $t_{A}^{p_{A}}=\left(t_{A} t_{B} t_{C}\right)=-1$ \\
\hline $\mathrm{SU}^{\prime}\left(V_{\mathbb{C}}\right.$ & $K_{\mathbb{C}}^{\prime}$ & $\left\langle p_{A}, p_{B}, p_{C}\right\rangle^{\prime}$ & $s: i x u^{-1}$ & $s_{A}^{2}=1,\left(s_{A} s_{B}\right)^{p_{C}}=-1$ \\
\hline
\end{tabular}

\section{Statement of the theorem}

We shall need some facts concerning complex reflection groups, to be applied to the group $K^{\prime}$. These are better explained in a more general setting, as in [Bourbaki, 1968]. Let $V$ be any finite-dimensional complex vector space, $G$ a finite subgroup of GL $(V)$ generated by reflections acting on $V$ on the left. Let $S$ be the algebra of polynomial functions on $V$. The group $G$ acts on $S$ on the right via $f g=f \circ g$. In addition there is the natural action of $\mathbb{C}^{\times}$on $V$ and on $S$, giving a representation of $\mathbb{C}^{\times} \times G$ on $S$. The $\mathbb{C}^{\times}$-stable subspaces $M$ are the graded ones and have a formal $\mathbb{C}^{\times}$-character, the Poincaré series $P_{M} \in \mathbb{C} \llbracket t \rrbracket$ of $M$ [Bourbaki, 1968, p103]. If the subspace $M$ is stable under $G$ as well, then it has a formal $\mathbb{C}^{\times} \times G$ character $P_{M} \in \mathbb{C} \llbracket t \rrbracket \otimes \mathrm{Ch}(G)$ : if the variable $t$ is identified with the standard character of $\mathbb{C}^{\times}$then $P_{M}=\sum m_{i k} \chi_{i} t^{k}$ where $m_{i k}$ is the multiplicity of the irreducible character $\chi_{i}$ of $G$ in the space homogenous polynomials of degree $k$ in $M$. $P_{M}$ can be viewed as a formal power series with characters of $G$ as coefficients or as a sum of characters of $G$ with formal power series as coefficients. The Poincaré series $P_{S}$ of the symmetric algebra $\mathrm{S}\left(V^{*}\right)$ and $P_{\Lambda}$ of the exterior algebra $\Lambda\left(V^{*}\right)$ satisfy $P_{S}(t) P_{\Lambda}(-t)=1$. Furthermore $P_{\Lambda}(-t)=\sum(-1)^{k} \Lambda_{V}^{k} t^{k}$ is a polynomial and $P_{S}(t)=1 / P_{\Lambda}(-t)$ expanded as a formal power series in $t$.

Let $R$ the subalgebra of $G$-invariants in $S$. It is a polynomial algebra in $\operatorname{dim}(V)$ algebraically independent homogeneous generators, whose degrees $d_{i}$ are uniquely determined up to order. Its Poincaré series is $P_{R}(t)=\prod\left(1-t^{d_{i}}\right)^{-1}$, expanded as formal power series. For any $x \in V$ let $\mathfrak{m}_{x}$ be the ideal of $R$ vanishing at $x$ or equivalently on the orbit $G x$. The particular ideal $\mathfrak{m}_{0}$ of $R$ vanishing at 0 is graded 
as is the ideal $S \mathfrak{m}_{0}$ it generates in $S$. Let $F$ be a $\mathbb{C}^{\times} \times G$-stable complement of $S \mathfrak{m}_{0}$ in $S$. Then $S \approx R \otimes F$, which implies that

$$
S / S \mathfrak{m}_{x} \approx\left(R / R \mathfrak{m}_{x}\right) \otimes F \approx F
$$

for any $x$ in $V$; it is always a $G$-isomorphism, but a $\mathbb{C}^{\times} \times G$ isomorphism only for $x=0$. The formal $\mathbb{C}^{\times} \times G$-character of $S=R \otimes F$ evidently satisfies $P_{S}(t)=$ $P_{R}(t) P_{F}(t)$. This equation can be written as $P_{S}(t)^{-1} P_{F}(t)=P_{R}(t)^{-1}$ i.e.

$$
\left(\sum(-1)^{k} \Lambda_{V}^{k} t^{k}\right) P_{F}(t)=\prod\left(1-t^{d_{i}}\right) .
$$

It is an equation for $P_{F}(t)$ in $\mathbb{C} \llbracket t \rrbracket \otimes \mathrm{Ch}(G)$. Specializing now to the case $G=$ $K^{\prime}, V \approx \mathbb{C}^{2}$ this equation reads

$$
\left(1-\operatorname{tr}_{V} t+\operatorname{det}_{V} t^{2}\right) P_{F}(t)=\left(1-t^{d_{1}}\right)\left(1-t^{d_{2}}\right) .
$$

The determinant $\operatorname{det}_{V}$ is \pm 1 on $K^{\prime}$ and after restriction of characters to $K$ it becomes $\operatorname{det}_{V} \equiv 1$. The multiplication by $\operatorname{tr}_{V}$ in $\mathrm{Ch}(K)$ is given by McKay's matrix $M=\left(m_{i j}\right)$ in the basis $\left\{\chi_{i}\right\}$, i.e. the incidence matrix of the graph in $\S 2$. The degrees $d_{1}, d_{2}$ may be found from the fact that $d_{1} d_{2}$ is the order of $K^{\prime}$ and $\left(d_{1}-1\right)+\left(d_{2}-1\right)$ the number of reflections [Bourbaki, 1968, p110,111] or located in the tables of Shephard and Todd [1954]. We list them here for reference.

\begin{tabular}{|l|l|l|l|l|l|}
\hline$K^{\prime}$ & $\mathrm{A}_{n}$ & $\mathrm{D}_{n}$ & $\mathrm{E}_{6}$ & $\mathrm{E}_{7}$ & $\mathrm{E}_{8}$ \\
\hline$d_{1}$ & $n+1$ & $2 n-2$ & 8 & 12 & 20 \\
\hline$d_{2}$ & 2 & 4 & 6 & 8 & 12 \\
\hline
\end{tabular}

Expand $P_{F}(t)=\sum \chi_{i} P_{i}(t)$ in terms of the irreducible characters $\chi_{i}$ of $K$. The equation for $P_{F}(t)$ becomes a system of linear equations for the unknown polynomials $P_{i}(t)$ with coefficient matrix $M(t):=\left[1-m_{i j} t+t^{2}\right]$ :

$$
\sum_{j=0}^{l}\left(1-m_{i j} t+t^{2}\right) P_{i}(t) \chi_{i}=\left(1-t^{d_{1}}\right)\left(1-t^{d_{2}}\right) \chi_{0} .
$$

It may be solved for the polynomials $P_{i}(t)$ in a mechanical fashion. (A few lines of Maple code which will do the job can be found in $\S 5$ ).

The polynomials $P_{i}(t)$ have been tabulated in a number of places, for example in the papers of Gonzales-Sprinberg and Verdier [1983] and of Kostant [1985]. A closed formula is given by Cramer's rule:

$$
P_{i}(t)=\frac{M_{i}(t)}{\operatorname{det} M(t)} .
$$

$M_{i}(t)$ is obtained from $M(t)$ by replacing the 'column' $\chi_{j}$ by the 'column' (1$\left.t^{d_{1}}\right)\left(1-t^{d_{2}}\right) \chi_{0}$. The determinant is $\operatorname{det} M(t)=\operatorname{det}\left(1-(2 I-C) t+t^{2}\right)$ which can be evaluated by the formula for the characteristic polynomial of a Cartan matrix [Bourbaki, Ch.V, §6,\#3]. Another formula is given by Kostant [1985, Theorem 1.11], which is particularly interesting because of the way it brings in the ADE root system and its Coxeter transformation: it gives the polynomials in the form $P_{i}(t)=\sum_{\varphi \in \Phi_{i}} t^{n(\varphi)}$ with $\varphi$ running over a set $\Phi_{i}$ of roots and equipped with a natural length function $n(\varphi)$, both defined in terms of the Coxeter element in the Weyl group. A different characterization of the $P_{i}(t)$ in terms of Hecke algebras can be found in [Springer 1985, §8.2]; it has its origin in [Lusztig, 1983] and plays a prominent role in [Lusztig, 1999]. These polynomial polynomials are related to the characters of $K$ as follows. 
Theorem 3.1. Let $\chi_{i}$ be the irreducible character associated to the node $i$ on the $A D E$ graph and let $e^{n \frac{\pi}{p} J}$ be the element of the maximal abelian subgroup associated to the node $n$ on a branch. The value of $\pi_{i}:=\chi_{i}+\bar{\chi}_{i}$ at $e^{n \frac{\pi}{p} J}$ is

$$
\pi_{i}\left(e^{n \frac{\pi}{p} J}\right)=P_{i}\left(e^{n \frac{\pi}{p} i}\right)
$$

obtained by the substituting $t=e^{n \frac{\pi}{p} i}$ into the polynomial $P_{i}(t)$

We add some remarks. Since every element of $K$ is conjugate to an element of some maximal abelian subgroup $T$ this formula gives all character values. While $\pi_{i} \equiv \chi_{i}+\bar{\chi}_{i}$ itself is not irreducible, the irreducible characters $\chi_{i}$ themselves can easily be extracted as well. This is clear if $\bar{\chi}_{i}=\chi_{i}$, i.e. if the corresponding node on the graph is fixed by the inversion involution, as must always be the case unless the node belongs to a coupled pair labeled $\pm n, \pm n$ on graph. The cases remaining occur only for odd $\mathrm{D}_{n}$ and for $\mathrm{E}_{6}$. Then one can use the following rule for the splitting of $P_{i}=P_{i}^{+}+P_{i}^{-}$which induces the splitting $\pi_{i} \equiv \chi_{i}+\bar{\chi}_{i}$ of the character. All polynomials $P_{i}(t)$ are of the form

$$
P_{i}(t)=\sum_{-h_{i}<k<h_{i}} t^{h_{i}+k}
$$

The splitting $P_{i}=P_{i}^{+}+P_{i}^{-}$is given by splitting the sum symmetrically at $k=0$.

\section{Proof of the theorem}

We start in a slightly more general setting. Let $V$ be a 2-dimensional unitary space, $G$ any finite subgroup of $\mathrm{U}(V)$. Let $S$ be the ring of polynomial functions on $V, L$ its quotient field of rational functions. Let $K, R$ be the invariants of $G$ in $L, S$. Consider the extension $S \supset R$ of integral domains. The classical ramification theory of prime ideals in extensions of Dedekind domains [Hecke, 1923, Kap.V] is not immediately applicable, since $L$ is not an algebraic function field, having transcendence degree 2 over $\mathbb{C}$. But if we pass from $V \approx \mathbb{C}^{2}$ to $(V-\{0\}) / \mathbb{C}^{\times} \approx \mathbb{P}^{1}$ by considering only $\mathbb{C}^{\times}$-invariant (= homogeneous) ideals of $S$, then the classical facts about the ramification of ideals in extensions of Dedekind domains remain applicable as far as needed. The details are spelled out in the following two lemmas.

Lemma 4.1. (a) The field extension $L \supset K$ is Galois with Galois group G. Let $\mathfrak{p}$ be a prime ideal in $R$. The prime ideals of $S$ over $\mathfrak{p}$ are permuted transitively by $G$ : they are of the form $\mathfrak{P}_{g}=\mathfrak{P} g, g \in H \backslash G$ where $H=H(\mathfrak{P})$ is the subgroup of $G$ leaving $\mathfrak{P}$ invariant.

(b) The field extension $L(\mathfrak{P}) \supset K(\mathfrak{p})$ of quotient fields for $S / \mathfrak{P} \supset R / \mathfrak{p}$ is Galois with Galois group $H / I$, where $I=I(\mathfrak{P})$ is the normal subgroup of $H$ fixing $S / \mathfrak{P}$.

Proof. These assertions are general facts [Bourbaki, 1985, Ch.V, $§ 2, n^{\circ} 2 ; 1968$, Ch.V, $\left.\S 5, n^{\circ} 5.\right]$.

Lemma 4.2. Assume that $\mathfrak{p}$ is a non-zero prime ideal of $R$ which ramifies in $S$, i.e. $\mathfrak{p}=\mathfrak{P} \cap R$ for some prime ideal $\mathfrak{P}$ of $S$ with $I(\mathfrak{P}) \neq 1$. Then

$(a) \mathfrak{P}=S a, a \in V^{*}$ a non-zero linear form.

(b)H is the subgroup of $G$ leaving the subspace $P=\{a=0\}$ invariant, $I$ its cyclic normal subgroup leaving $P$ pointwise fixed. 
(c) $S \mathfrak{p}$ decomposes as a product of ideals in the form

$$
S \mathfrak{p}=\prod_{g \in H \backslash G} \mathfrak{P}_{g}^{e}
$$

where $e$ is the order of $I$.

(d)The residue ring $S / \mathfrak{p}(:=S / S \mathfrak{p})$ decomposes as a direct product of rings

$$
S / \mathfrak{p} \approx \prod_{g \in H \backslash G} S / \mathfrak{P}_{g}^{e}
$$

and this decomposition exhibits the representation of $\mathbb{C}^{\times} \times G$ on $S / \mathfrak{p}$ as induced by the representation of $\mathbb{C}^{\times} \times H$ on $S / \mathfrak{P}^{e}$.

Proof. The assumption $I(\mathfrak{P}) \neq 1$ implies that the zero set $P$ of $\mathfrak{P}$ in $V$ is pointwise fixed by a linear transformation $\neq 1$. Since $V$ is 2 -dimensional it must be of the form $P=\{a=0\}$ for some linear form $a \in V^{*}$ and hence $\mathfrak{P}=S a$. This proves (a), and (b) is then clear [Bourbaki,1968, Ch.V, $\left.\S 5, n^{\circ} 5\right]$.

The ideal $\mathfrak{P}=S a$ is $\mathbb{C}^{\times}$-invariant and maximal among such ideals since $\operatorname{dim} V=$ 2. In particular, any two distinct ideals $\mathfrak{P}_{g}, \mathfrak{P}_{g^{\prime}}$ satisfy $\mathfrak{P}_{g}+\mathfrak{P}_{g^{\prime}}=S$. This implies the decompositions (c) and (d) of $S \mathfrak{p}$ and of $S / \mathfrak{p}$ [Bourbaki, 1961, Ch.II, $\S 1, n^{\circ} 2$ ]. The last assertion then follows from the definition of an induced representation [Serre, 1977, p28].

As they stand, the representations in the lemma are infinite dimensional: the representation spaces $S / \mathfrak{p}$ and $S / \mathfrak{P}_{g}^{e_{g}}$ are in fact modules over the group algebra with coefficients in the ring $R / \mathfrak{p}$. Finite-dimensional representations can be obtained as follows. In the setting of the lemma, part (b), let $\mathfrak{m}$ be a maximal ideal of $R$ containing $\mathfrak{p}$. It is the ideal of $R$ vanishing on the orbit $G x$ for some $x$ in the 1dimensional subspace $P=\{a=0\}$. Assume $x \neq 0$. Let $\mathfrak{M}_{g}$ be the maximal ideal of $S$ vanishing at $g x$. These ideals are no longer invariant under $\mathbb{C}^{\times}$. Consider however the group of elements $h \in G$ satisfying $h x=\lambda(h) x$ for some $\lambda(h) \in \mathbb{C}^{\times}$. Since $P$ is 1-dimensional, this group is the subgroup $H$ of $G$ leaving $P$ invariant. Let $I$ be the subgroup of $H$ leaving $P$ pointwise fixed. Then $\lambda: H / I \rightarrow \mathbb{C}^{\times}$is a multiplicative character of $H / I$. Its image is a cyclic subgroup $C$ of $\mathbb{C}^{\times}$.

The following corollary (which remains valid with $e=1$ if $\mathfrak{m}$ is unramified, i.e. not fixed by any nontrivial element of $G$ ) will give the desired reduction to finite dimensions.

Lemma 4.3. (a)The residue ring $S / \mathfrak{m}$ decomposes as a direct product

$$
S / \mathfrak{m}=\prod_{g \in I \backslash G} S / \mathfrak{M}_{g}^{e},
$$

and this decomposition exhibits the representation of $C \times G$ on $S / \mathfrak{m}$ as induced by the representation of $C \times I$ on $S / \mathfrak{M}^{e}$.

(b) The representation of $I$ on $S / \mathfrak{M}^{e}$ is its regular representation:

$$
S / \mathfrak{M}^{e} \approx \prod_{s \in I} \mathbb{C}_{s}
$$

(c) The representation of $G$ on $S / \mathfrak{m}$ is

$$
S / \mathfrak{m} \approx \sum_{\nu=0}^{e-1} \operatorname{In} d_{I}^{G} \epsilon^{\nu} .
$$


where $\epsilon$ is the character of $I$ on the line $\mathbb{C} a$ in $V^{*}$.

Proof. (a)Consider the representation $S \mathfrak{m}$ as an intersection of primary ideals in $S$. These are necessarily powers of the maximal ideals $\mathfrak{M}_{g}$ of $S$ vanishing at the points $x g, g \in G$. Since $S \mathfrak{p}=\prod_{g \in H \backslash G} \mathfrak{P}_{g}^{e}$ and since $I$ is the subgroup leaving $\mathfrak{M}$ invariant this decomposition is given by $S \mathfrak{m}=\prod_{g \in I \backslash G} \mathfrak{M}_{g}^{e}$. The direct product decomposition of $S / \mathfrak{m}$ then follows, as does its identification as an induced representation.

(b) The group $I$ consists of reflections in the subspace $P=\{a=0\}$ and may be considered as a reflection group on the quotient space $V / P$. The assertion then follows from general facts about reflection groups [Bourbaki 1968, p107]. More directly, the classes of $1, a, \cdots, a^{e-1}$ form a basis for $S / \mathfrak{M}^{e}$ and provide the decomposition of $S / \mathfrak{M}^{e}$ as a sum over the $e$ irreducible characters of the cyclic group $I$ characteristic of the regular representation:

$$
S / \mathfrak{M}^{e} \approx \sum_{\nu=0}^{e-1} \mathbb{C} a^{\nu}
$$

The components in this sum are the powers $\epsilon^{\nu}, \nu=0,1, \cdots, e-1$, of the character $\epsilon$ by which $I$ acts on the line $\mathbb{C} a$ in $V^{*}$.

(c)Follows from (2) and (4).

Now suppose $G \subset \mathrm{U}(V)$ is a normal extension of $K \subset \mathrm{SU}(V)$ as at the beginning of $\S 2$. Let $T$ be one of the three subgroups $T_{A}, T_{B}, T_{C}$ of $K$ and take for $x$ an eigenvector of $T$. The subgroup $H$ of $G$ satisfying $x h=\lambda(h) x$ is one of the three maximal abelian subgroups of $G$, namely the one which contains $T$. If the subgroup $I$ of $G$ fixing $x$ is non-trivial, then it is cyclic and its generator is a reflection $s$ which centralizes $T$. The decomposition $V=P^{+}+P^{-}$of $V$ into eigenspaces of $T$ produces the situation of case (1) in $\S 2$ :

$$
T:\left(\begin{array}{cc}
\lambda & 0 \\
0 & \lambda^{-}
\end{array}\right), \quad s=\left(\begin{array}{cc}
1 & 0 \\
0 & \epsilon
\end{array}\right) .
$$

Here $x$ belongs to $P^{+}$and now $\epsilon=\operatorname{det} \mid I$. The decomposition (3) becomes

$$
S / \mathfrak{m} \approx \sum_{\nu=0}^{e-1} \operatorname{det}^{\nu} \otimes \operatorname{Ind}_{I}^{G} 1, \quad \operatorname{Ind}_{I}^{G} 1 \approx \prod_{g \in I \backslash G} S / \mathfrak{M}_{g}
$$

the second isomorphism coming from $S / \mathfrak{M}_{g} \approx \mathbb{C}$. This equation remains also valid when $I=1$. Furthermore, under the homomorphism $\lambda: H \rightarrow C$ the action of $C$ by scalar multiplications agrees with that of $H$ by permutations of cosets $I g \mapsto h I g=I h g$, but from the opposite side of $G$. This follows from the relation $x h g=\lambda(h) x g$.

Assume further that $G$ is generated by reflections. Then $S$ is free over $R$, say $S \approx R \otimes F$ as a tensor product over $\mathbb{C}$. Then $S / \mathfrak{m} \approx(R / \mathfrak{m}) \otimes F$ and $R / \mathfrak{m} \approx \mathbb{C}$ leads to

$$
S / \mathfrak{m} \approx F .
$$

If the subspace $F$ of $S$ is chosen to be stable under $\mathbb{C}^{\times} \times G$ then this is a $\mathbb{C}^{\times} \times G$ isomorphism and the properties concerning the representation of $C \times G$ on $S / \mathfrak{m}$ carry over to $F$. 
At this point we return to the group $K^{\prime}$ in the role of $G$. Thus $S \approx R \otimes F$ and the representation of $K^{\prime}$ on $F$ is equivalent to its regular representation in the direct product of copies $\mathbb{C}_{g}$ of $\mathbb{C}$,

$$
F \approx \prod_{g \in K^{\prime}} \mathbb{C}_{g}
$$

The elements of the direct product are functions $f_{g}$ of $g \in K^{\prime}$ on which $K^{\prime}$ acts by translations in the variable $g$ from the left: $f_{g} \mapsto f_{l^{-1} g}$. This representation of $K^{\prime}$ extends to the biregular representation of $K^{\prime} \times K^{\prime}$ by left-right translations $f_{g} \mapsto f_{l^{-1} g r}$.

Lemma 4.4. The biregular representation of $K^{\prime} \times K^{\prime}$ decomposes on $K \times K$ in the form

$$
\sum \chi_{i} \otimes \pi_{i}, \quad \pi_{i}:=\bar{\chi}_{i}+\chi_{i}
$$

The sum runs over the irreducible characters $\chi_{i}$ of $K$.

Proof. Consider the decomposition of the biregular character of $K^{\prime} \times K^{\prime}$ into two pieces corresponding to the decomposition of functions on $K^{\prime}=K \cup s K$ into functions supported on one of the two cosets. That the functions supported on $K$ give the regular representation $\sum_{i} \chi_{i} \otimes \bar{\chi}_{i}$ is clear. That those on $s K$ give $\sum \chi_{i} \otimes \chi_{i}$ follows from the fact that $K^{\prime} / K$ acts by $\chi \mapsto \bar{\chi}$ on the characters of $K$ : the map $f_{k} \mapsto f_{s k}$ sends function on $K$ to functions on $s K$, transforming the left action $f_{l k}$ of $l \in K$ into the twisted action $f_{s\left(s^{-1} l s\right) k}$.

Remark. The decomposition of the lemma can also be understood as follows. An irreducible character $\chi^{\prime}$ of $K^{\prime}$ either remains irreducible on $K$ or else decomposes into two a pair of characters conjugate under $K^{\prime} / K$. All irreducible characters of $K$ occur twice in this way: if $\chi^{\prime}=\bar{\chi}^{\prime}$ then twice in this $\chi^{\prime}$; if $\chi^{\prime} \neq \bar{\chi}^{\prime}$ then once in $\chi^{\prime}$ and once in $\bar{\chi}^{\prime}$. These facts follow from general properties of subgroups of index 2 [Weyl, 1939, p.159]. They can also be found in [Gonzales-Sprinberg and Verdier, 1983].

Compare the representation (5) of $C \times K^{\prime}$ on $S / \mathfrak{m} \approx F$ with the decomposition (6) of the biregular representation of $K^{\prime} \times K^{\prime}$. In (5) the group $K^{\prime}$ acting on $S / \mathfrak{m} \approx F$ corresponds to the subgroup $K^{\prime} \times 1$ of $K^{\prime} \times K^{\prime}$ acting on $\prod_{g \in K^{\prime}} \mathbb{C}_{g}$. The character of $K$ in (5) corresponds to character of $K \times 1$ in (7), obtained by evaluating the right factors $\pi_{i}$ in the lemma at the identity element. The action of $1 \times K$ in (7) is not directly visible in (5). However, the action of the subgroup $1 \times T$ is visible in (5) and corresponds to the action of the subgroup $C$ of $\mathbb{C}^{\times}$via the homomorphism $\lambda: T \rightarrow C$, as remarked after (5). The group $T$ consists of elements $e^{n \frac{\pi}{p} J}$ where $J \in \mathbb{H}$ satisfies $J^{2}=-1$. Its eigenspaces are those of $J$ acting on $V \approx \mathbb{H}$ by right multiplication with eigenvalues $\pm i$. Choose $x$ in the $+i$ eigenspace, so that $x J=i x$. Then $x e^{n \frac{\pi}{p} J}=e^{n \frac{\pi}{p} i} x$, i.e. $\lambda\left(e^{n \frac{\pi}{p} J}\right)=e^{n \frac{\pi}{p} i}$. The result is that under the isomorphism (6) the character of $K \times T$ coming from the biregular representation of $K^{\prime} \times K^{\prime}$ on $\prod_{g \in K^{\prime}} \mathbb{C}_{g}$ agrees with the character of $K \times C$ coming from the character of $K \times \mathbb{C}^{\times}$on $F$, i.e.

$$
\sum P_{i}\left(e^{n \frac{\pi}{p} i}\right) \chi_{i}=\sum \pi_{i}\left(e^{n \frac{\pi}{p} J}\right) \chi_{i}
$$

Comparison of the coefficients of $\chi_{i}$ concludes the proof of the theorem. 


\section{An EXAMPLE}

We list the polynomials $P_{i}(t)$ and the character values $\chi_{i}\left(e^{n \frac{\pi}{p} J}\right)$ for the binary icosahedral group $K=\langle 5,3,2\rangle$ of type $\mathrm{E}_{8}$. The labels $1 A, 2 A, 3 A, 4 A$, * refer to the 5 nodes on branch $A$, the node * being common to all branches.

$$
\begin{aligned}
& P_{1 A}(t)=t+t^{11}+t^{19}+t^{29} \\
& P_{2 A}(t)=t^{2}+t^{10}+t^{12}+t^{18}+t^{20}+t^{28} \\
& P_{3 A}(t)=t^{3}+t^{9}+t^{11}+t^{13}+t^{17}+t^{19}+t^{21}+t^{27} \\
& P_{4 A}(t)=t^{4}+t^{8}+t^{10}+t^{12}+t^{14}+t^{16}+t^{18}+t^{20}+t^{22}+t^{26} \\
& P_{*}(t)=\sum_{k=0}^{5} t^{15-2 k}+t^{15+2 k} \\
& P_{2 B}(t)=t^{6}+t^{8}+t^{12}+t^{14}+t^{16}+t^{18}+t^{22}+t^{24} \\
& P_{1 B}(t)=t^{7}+t^{13}+t^{17}+t^{23} \\
& P_{1 C}(t)=t^{6}+t^{10}+t^{14}+t^{16}+t^{20}+t^{24} \\
& P_{0}(t)=1+t^{30}
\end{aligned}
$$

Maple will compute these polynomials if instructed this way:

$$
\begin{aligned}
& >\text { with(linalg): } \\
& >\mathrm{C}:=\operatorname{matrix}([[2,-1,0,0,0,0,0,0,0],[-1,2,-1,0,0,0,0,0,0],[0,-1,2,-1,0,0,0,0,0], \\
& {[0,0,-1,2,-1,0,0,0,0],[0,0,0,-1,2,-1,0,0,0],[0,0,0,0,-1,2,-1,0,-1],} \\
& [0,0,0,0,0,-1,2,-1,0],[0,0,0,0,0,0,-1,2,0],[0,0,0,0,0,-1,0,0,2]]) ; \\
& >\mathrm{A}:=1+\mathrm{t}^{\wedge} 2-\mathrm{t}^{*}(2-\mathrm{C}) \\
& >\mathrm{B}:=\left(\left(1-\mathrm{t}^{\wedge} 12\right)^{*}\left(1-\mathrm{t}^{\wedge} 20\right)\right)^{*} \operatorname{vector}([1,0,0,0,0,0,0,0,0]) ; \\
& >\mathrm{P}:=\operatorname{linsolve}(\mathrm{A}, \mathrm{B}) ; \\
& >\operatorname{sort}(\operatorname{expand}(\mathrm{P}[4]))
\end{aligned}
$$

The characters are $\pi_{i}=2 \chi_{i}$ and the values $\chi_{i}\left(e^{n \underline{\pi} J}\right)$ are listed in the following table. Both $i=0$ and $n=0$ are omitted. Notation:

$$
\begin{aligned}
& \tau:=\frac{1+\sqrt{5}}{2}=e^{\frac{\pi \mathrm{i}}{5}}+e^{-\frac{\pi \mathrm{i}}{5}}=1+e^{\frac{2 \pi \mathrm{i}}{5}}+e^{-\frac{2 \pi \mathrm{i}}{5}}, \\
& \tau^{-}:=\frac{1-\sqrt{5}}{2}=e^{\frac{3 \pi \mathrm{i}}{5}}+e^{-\frac{3 \pi \mathrm{i}}{5}}=1+e^{\frac{4 \pi \mathrm{i}}{5}}+e^{-\frac{4 \pi \mathrm{i}}{5}} \\
& {\left[(x-\tau)\left(x+\tau^{-}\right)=x^{2}-x-1\right] .}
\end{aligned}
$$

\begin{tabular}{|c|c|c|c|c|c|}
\hline & $e^{ \pm \frac{\pi}{5} J_{A}}$ & $e^{ \pm \frac{3 \pi}{5} J_{A}}$ & $e^{*}=-1$ & $e^{n \frac{\pi}{3} J_{B}}$ & $e^{\frac{\pi}{2} J_{C}}$ \\
\hline$\chi_{1 A}$ & $\tau$ & $\tau^{-}$ & -2 & $(-1)^{n+1}$ & 0 \\
\hline$\chi_{2 A}$ & $\tau$ & $\tau^{-}$ & +3 & 0 & -1 \\
\hline$\chi_{3 A}$ & 1 & 1 & -4 & \pm 1 & 0 \\
\hline$\chi_{4 A}$ & 0 & 0 & +5 & -1 & 0 \\
\hline$\chi_{*}$ & -1 & 0 & +6 & 0 & $i$ \\
\hline$\chi_{1 B}$ & $\tau^{-}$ & $\tau$ & -2 & $(-1)^{n+1}$ & 0 \\
\hline$\chi_{2 B}$ & 1 & -1 & +4 & 1 & 0 \\
\hline$\chi_{1 C}$ & $\tau^{-}$ & $\tau$ & +3 & 0 & -1 \\
\hline
\end{tabular}

\section{REMARKS ON INVARIANTS AND GEOMETRY}

Invariants. The proof of the theorem was given in the abstract setting of ramification of prime ideals, which seemed clearest and perhaps useful in other situations. It is possible, however, to give a description of the invariants of a finite subgroup $G$ of $\mathrm{U}(2)$ which makes the $\operatorname{ring} R$ and the ramification of its homogenous prime ideals in $S$ more explicit. Let $P$ be the 1-dimensional subspace of $V \approx \mathbb{C}^{2}$, and let $a$ be a linear form defining it as $P=\{a=0\}$. Let $H$ and $I$ the subgroups of 
$G$ leaving $P$ invariant and pointwise fixed. If $h \in H$ then $a h=\lambda_{P}(h) a$ for some multiplicative character $\lambda_{P}: H \rightarrow \mathbb{C}^{\times}$. Let

$$
f_{P}(x):=\prod_{g \in[H \backslash G]} a(g x),
$$

the product being taken over a set of coset representative denoted $[H \backslash G]$. Under the left action $x \mapsto l x$ by $l \in G$ the polynomial $f_{P}(x)$ transforms according to the rule

$$
f_{P}(l x)=\mu_{P}(l) f_{P}(x), \quad \mu_{P}:=\left(\lambda_{P}\right)^{|H \backslash G|},
$$

showing that $f_{P}$ is a relative invariant of $G$. The zero-set $\left\{f_{P}=0\right\}$ is $G P$, the union of the lines $P_{g}:=g P, g \in G / H$. Fix an affine line $x=a z+b$ in $V$ and let $z_{P}$ be its point of intersection with $P$. The $P_{g}$ then become the roots $z_{g}$ of the equation $f_{P}(z)=0$ which represents $f_{P}=0$. Now consider any relatively invariant form $f$ on $V$. The equation $f=0$ defines a set of points on $\mathbb{P}(V)$ invariant under $G$. If one expresses this set as a union of $G$-orbits $\bigcup_{P} G P$, each counted with the multiplicity $m_{P}$ of $z_{P}$ as a root of $f(z)=0$, then the polynomial $\prod_{P} f_{P}^{m_{P}}(z)$ has the same zeros and multiplicity as $f(z)$, hence differs from $f$ by a constant factor. As form on $V, f=\prod_{P} f_{P}^{m_{P}}$ is an absolute invariant if the multiplicative character $\prod_{P} \mu_{P}^{m_{P}}$ is $=1$. In the case when $G$ is a finite subgroup $K=\left\langle p_{A}, p_{B}, p_{C}\right\rangle$ of SU(2) one obtains generators for $R$ in this way by restricting $P$ to run over the eigenspace $P_{A}, P_{B}, P_{C}$ of the three maximal abelian subgroups $T_{A}, T_{B}, T_{C}$ of $G$. In case $G$ is the reflection group $K^{\prime}=\left\langle p_{A}, p_{B}, p_{C}\right\rangle^{\prime}$ two of such invariants suffice as algebraically independent generators for $R$. The decomposition $S \mathfrak{f}=\prod \mathfrak{F}_{g}^{e}$ of an ideal $\mathfrak{f}=R f$ generated by such an invariant $f=\prod_{P} f_{P}^{m_{P}}$ is clear.

A question arises at this point. The discussion here, and the proof of the theorem by reduction to ramification of primes in a Dedekind domain, depends heavily on $\operatorname{dim} V=2$. The question is whether the decomposition $S \mathfrak{p}=\prod \mathfrak{P}_{g}^{e}$ nevertheless remains valid if $G$ is a reflection group in arbitrary dimension and $\mathfrak{P}$ the ideal vanishing on an arbitrary intersection of reflecting hyperplanes.

Geometry. The discussion of the ramification of primes in the ring of invariants proceeded in the language of commutative algebra. Another perspective emerges if it is rephrased in the language of algebraic geometry. The translation runs as follows. The rings $S$ and $R$ are the regular functions on the affine varieties $V$ and $Q:=G \backslash V$, the inclusion $S \supset R$ being the pull-back via the quotient map $\pi: V \rightarrow Q$. These varieties come with actions of the multiplicative group $\mathbb{C}^{\times}$whose quotients give projective varieties $\bar{V} \approx \mathbb{P}^{1}$ and $\bar{Q}$ together with a map $\bar{\pi}: \bar{V} \rightarrow \bar{Q}$. $V$ and $Q$ are affine algebraic surfaces, $\bar{V}$ and $\bar{Q}$ projective curves. The maps $\pi$ and $\bar{\pi}$ are ramified coverings.

A 1-dimensional subspace $P$ of $V$ corresponds to a point $\bar{P}$ of $\bar{V}$ and its image $p$ in $Q$ to a point $\bar{p}$ in $\bar{Q}$. If $\mathfrak{p}$ is the ideal of $R$ defining the image $p=\pi(P)$ of $P$ in $Q$, then $S \mathfrak{p}$ is the ideal of $S$ defining the inverse image $\pi^{-1}(p)=G P=\bigcup g P$ in $V$. The factorization $S \mathfrak{p}=\prod_{g \in I \backslash G} \mathfrak{P}_{g}^{e}$ corresponds to the decomposition of $\pi^{-1}(p)$ into the individual lines $g P$ each counted with multiplicity $e . S / \mathfrak{p}$ is the ring of 'functions' on $\pi^{-1}(p)$ and $S / \mathfrak{p}=\prod_{g \in I \backslash G} S / \mathfrak{P}_{g}^{e}$ is its decomposition into functions supported on the individual lines $g P$. If $P$ is the replaced by a point $x$ in it, then $S \mathfrak{m}=\prod_{g \in I \backslash G} \mathfrak{M}_{g}^{e}$ defines the decomposition of the fiber $\pi^{-1} \pi(x)=G x$ into multiple points and $S / \mathfrak{m}=\prod_{g \in I \backslash G} S / \mathfrak{M}_{g}^{e}$ the decomposition of the ring of 'functions' on the orbit Gxinto functions supported at its individual points $g x$. 
The isotropy group $I$ of the base-point $x$ is normal in the group $H$ leaving $P$ invariant and the groups $G$ and $H$ act on opposite sides on the orbit: if $G$ acts on $G x \approx G / I$ on the left, then $H$ acts on right via $(g x) h:=g h x$. The difficulty with this picture comes from the fact that the points $g x$ in an orbit may have higher multiplicity, accounting for the fact that the 'functions' take on values in the rings $S / \mathfrak{M}_{g}^{e}$ depending on the points, rather than in the field $S / \mathfrak{M}_{g}=\mathbb{C}$. It is for this reason that the algebraic description seems preferable.

Nevertheless, the interpretation of ramification as representing fiber decomposition with multiplicity, i.e.

$$
S \mathfrak{m}=\prod \mathfrak{M}_{g}^{e} \quad \text { represents } \quad \pi^{-1}[\pi(x)]=\sum e\left[x_{g}\right],
$$

is highly suggestive: it suggests a realization of the representation of $G$ with character $\pi_{i}$ by monodromy in this fiber system, with $G$ acting on a fiber of the map $\pi: V \rightarrow Q$ by continuous transport around loops in the set of regular values. Of particular interest in this connection is the different ideal of $S$ over $R$ [Zariski and Samuel, 1958, Ch.V, §11]. In geometric terms it is the ideal $\mathfrak{D}$ of $S$ defining the ramification locus, i.e. the inverse image of the set of critical values of $\pi: V \rightarrow Q$. Equivalently, $\mathfrak{D}$ is the ideal in the homogenous coordinate $\operatorname{ring} S$ of $\bar{V} \approx \mathbb{P}^{1}$ defining the ramification locus of the ramified covering $\bar{\pi}: \bar{V} \rightarrow \bar{Q}$ of compact Riemann surfaces. It factors as $\mathfrak{D}=\prod_{P} \mathfrak{P}^{m_{P}}$ where $\bar{P}$ runs over the points of $\bar{V}$ at which the Jacobian $D$ of $\bar{\pi}$ vanishes with multiplicity $m_{P} \neq 0$. It is in fact the principal ideal $\mathfrak{D}=S D$. In case $G$ is a reflection group (in arbitrary dimension) the ramification locus is the arrangement of reflecting hyperplanes and $D$ the product of the linear forms defining them. [Bourbaki, 1968, p.116].

In case $G$ is a finite subgroup of $\mathrm{U}(2)$, e.g. a Klein group, the ramification locus is the union of the singular lines $P$ which have the maximal abelian subgroups $H$ of $G$ as stabilizers. As mentioned in $\S 2$, each $H$ is the stabilizer of two singular lines $P, P^{-}$which may or may not be interchanged by the group $N(H) / H$, of order 2 or $1 . D \propto \prod_{P}\left(\alpha_{P}\right)^{m_{P}}$ is a constant multiple of the product of linear forms $a_{P}$ defining the singular lines, $a_{P}$ being taken with multiplicity $m_{P}=|\bar{H}|-1, \bar{H}:=$ the image of $H$ in $\overline{\mathrm{U}}(V):=\mathrm{U}(V) / \mathrm{U}(\mathbb{C})$. If the $\alpha_{P}$ s are grouped into $G$-orbits the product becomes

$$
D \propto f_{A}^{p_{A}-1} f_{B}^{p_{B}-1} \ldots
$$

Here $H_{A}, H_{B}, \cdots$ runs over a complete system of representatives for the conjugacy classes of $H \mathrm{~s}, f_{A}=\prod_{g \in\left[G / H_{A}\right]}\left(a_{A} g\right)$, and $p_{A}=\left|\bar{H}_{A}\right|$.

On the other hand, the Jacobian is $D \propto \partial\left(f_{1}, f_{2}\right) / \partial\left(z_{1}, z_{2}\right)$ for any are linear coordinates $z_{1}, z_{2}$ of $V$ and any two generic forms $f_{1}, f_{2}$ of the type $f_{P}$, for which $\bar{H}=$ $\{1\}$. If one compares degrees in the relation $\partial\left(f_{1}, f_{2}\right) / \partial\left(z_{1}, z_{2}\right) \propto f_{A}^{p_{A}-1} f_{B}^{p_{B}-1} \ldots$ one finds

$$
2(|\bar{G}|-1)=\frac{2|\bar{G}|}{|N(\bar{H}) /| \bar{H} \mid}(|\bar{H}|-1)+\cdots .
$$

One thus returns to the class equation for subgroups of $\mathrm{U}(2)$, the starting point for their classification. The discussion is based on [Klein, 1884, Ch.5, §2].

\section{REFERENCES}

[1] N. Bourbaki, Algèbre commutative. Chapitres 1 et 2. Hermann, Paris, 1961.

[2] N. Bourbaki, Groupes et algèbres de Lie. Chapitres 4,5 et 6. Hermann, Paris, 1968.

[3] N. Bourbaki, Algèbre commutative. Chapitres 5,6 et 7. Hermann, Paris, 1985. 
[4] H.S.M. Coxeter, Regular Complex Polytopes. Second Edition. Cambridge University Press, 1974.

[5] G. Gonzales-Sprinberg and J.-L. Verdier, Construction géométrique de la corrrespondence de McKay. Ann. Sci. Ecole Norm. Sup. t.16, nº3, 410-449(1983).

[6] E. Hecke. Vorlesungen über die Theorie der Algebraischen Zahlen. Leipzig 1923. Reprinted by Chelsea Publishing Co., New York, 1970.

[7] F. Klein. Vorlesungen über das Ikosaeder und die Auflösung der Gleichung vom fünften Grade. Teubner, Leipzig, 1884.

[8] H. Knörrer. Group representations and the resolution of rational double points. In: Finite groups - Coming of Age. Proceedings, Montreal 1982 (J. Mckay, ed.). Contemporary Math., v.45, AMS, 175-222, Providence, 1985.

[9] B. Kostant, The McKay correspondence, the Coexeter element, and representation theory. In: Élie Cartan el les mathémaiques d'aujourd'hui (Lyon, 1984). Astérisque, Hors séries, 209-255 (1985).

[10] G. Lusztig, Some examples of square integrable representations of semisimple p-adic groups. Trans. AMS 277, 153-215 (1983).

[11] G. Lusztig, Subregular nilpotent elements and bases in K-theory. Cand. J. Math. 51(6), 11941225 (1999).

[12] J. McKay. Graphs, singularities, and finite groups. AMS, Proc. Symp. Pure Math. Vol. 37, 183-186, 1980.

[13] J.P. Serre. Linear Represenations of Finite Groups. Springer Verlag, New York, 1977.

[14] G.C. Shephard and J.A. Todd. Finite unitary reflection groups. Can. J. Math. 6, 111-135 (1954).

[15] T.A. Springer, Poincaré series of binary polyhedral groups and McKay's correspondence. Math. Ann. 278, 587-598 (1985).

[16] R. Steinberg, Finite subgroups of $\mathrm{SU}_{2}$, affine Dynkin diagrams and affine Coxeter elements. Pac. J. Math. 118, 587-598 (1985). Preprint 1982.

[17] H. Weyl. The Classical Groups. Their invariants and Representations. Second Edition. Princeton University Press, Princeton, 1939.

[18] O. Zariski and P. Samuel. Commutative Algebra I. D. Van Nostrand Company, Inc. Princeton, 1958.

Department of Mathematics, University of Ottawa, Ottawa, Cananda

E-mail address: rossmann@uottawa.ca 